\title{
Cross-over study of influence of oral vitamin C supplementation on inflammatory status in maintenance hemodialysis patients
}

KunYing Zhang ${ }^{1,2,3}$, YinHui Li $i^{5}$, XuYang Cheng ${ }^{1,2,3}$, Li Liu ${ }^{1,2,3}$, WenYing Bai ${ }^{6}$, WeiYa Guo ${ }^{7}$, LeiYun Wu ${ }^{8}$ and Li ZuO ${ }^{1,2,3,4^{*}}$

\begin{abstract}
Background: Both vitamin C deficiency and inflammation are prevalent in maintenance hemodialysis (MHD) patients. In this study, we aimed to elucidate the effect of oral vitamin C supplementation on inflammatory status in MHD patients with low vitamin C level and high hypersensitive C-reactive protein (hs-CRP) level.

Methods: A total of 128 patients were recruited in our present study. Patients were divided into two groups. In group 1 ( $n=67$ ), patients were orally administered with $200 \mathrm{mg} /$ day vitamin $C$ in the first 3 months, and then the vitamin C supplementation was withdrawn in the next 3 months. In group $2(n=61)$, patients were not given vitamin $C$ in the first 3 months, and then they were orally administered with $200 \mathrm{mg} /$ day in the next 3 months. Levels of hs-CRP, prealbumin, albumin and hemoglobin as well as the EPO resistance index (ERI) were determined at the baseline and every 3 months throughout the study. Plasma vitamin C level was determined by high-performance liquid chromatography with UV detection.
\end{abstract}

Results: Among the 128 patients, 28 of them dropped out of the study before completion. Consequently, a total of 100 patients (group 1: $n=48$; group 2: $n=52$ ) were included in the final analysis. At the baseline, the plasma vitamin C level of all patients was less than $4 \mu \mathrm{g} / \mathrm{mL}$. However, this proportion was decreased to $20 \%$ after the vitamin C supplementation for 3 months. Compared with patients without the vitamin C supplementation, a decreased level of hs-CRP and an increased level of prealbumin were induced by the vitamin $C$ supplementation for 3 months in both groups. However, levels of these biomarkers returned to their original state after the supplementation was withdrawn. Same beneficial effects on plasma albumin, hemoglobin and ERI response to vitamin C supplementation were observed in the two groups without statistical significance.

Conclusions: The inflammatory status in MHD patients with plasma vitamin C deficiency and high levels of inflammatory markers could be partially improved by long-term oral administration of small doses of vitamin C.

Trial registration: The clinical trial number: NCT01356433.

Keywords: Hemodialysis, Inflammation, Vitamin C

\footnotetext{
*Correspondence: ZuoLiMD@Gmail.com

'Department of Medicine, Renal Division, Peking University First Hospital, Beijing, P. R. China

${ }^{2}$ Peking University Institute of Nephrology, Beijing, P. R. China

Full list of author information is available at the end of the article
} 


\section{Background}

The life expectancy of maintenance hemodialysis (MHD) patients is significantly lower than that of healthy subjects [1]. Atherosclerotic cardiovascular disease is the leading cause of death in this population. In addition to traditional cardiovascular risk factors, there are some non-traditional risk factors, including micro-inflammation [2,3]. Therefore, measures should be taken to correct the conditions with a possibly negative effect on arthrosclerosis.

Inflammation is prevalent in MHD patients. As an inflammatory marker, C-reactive protein (CRP) is an important sensitive acute phase reactant [4]. Previous investigations demonstrated that high level of serum CRP is a strong predictor of cardiovascular mortality both in healthy subjects [5] and MHD patients [6].

Vitamin $C$ is one of the most important water soluble antioxidants. It is well established that the plasma vitamin $\mathrm{C}$ level is generally lower in MHD patients $[7,8]$ than that in general population, which is attributed to inadequate dietary intake, oxidative stress [9] and loss during dialysis session $[7,10]$.

The low level of plasma vitamin $\mathrm{C}$ as well as inflammatory status has been recently reported to be closely related to the increased risk of cardiovascular morbidity and mortality in either MHD or peritoneal dialysis (PD) patients $[6,11]$. Our previous cross-sectional analysis showed that low level of plasma vitamin $C$ is negatively associated with the CRP level [12].

The hypothesis that the inflammatory status may be improved by vitamin $C$ supplementation has been studied in a limited number of investigations based on a limited number of patients, resulting in conflicting results. One study was conducted on 33 MHD patients for 2 months [13], another one was conducted on $20 \mathrm{MHD}$ patients for 2 months [14], and both studies did not get positive conclusions. However, an investigation documented that the 8-hydroxy-2'-deoxyguanosine (8-OHdG) level of cellular DNA is reduced after the vitamin $C$ supplementation for 8 weeks in chronic hemodialysis patients [15]. These previous conflicting results might be partly due to either limited sample size or short period of observation.

In the present study, we designed a randomized controlled cross-over study with relatively large sample size and aimed to investigate the effect of vitamin $C$ supplementation on inflammatory status in MHD patients.

\section{Methods}

\section{Study patients}

The effect of oral vitamin C supplementation on inflammatory status in MHD patients with low vitamin C level and high hypersensitive CRP (hs-CRP) level was investigated using a randomized controlled cross-over study. Patients who met all of the following inclusion criteria were included: (1) more than 18 years old; (2) under stable condition, receiving conventional hemodialysis for 4-4.5 hours thrice weekly and MHD for at least 3 months; Kt/V > 1.2; (3) plasma vitamin C level $<4 \mu \mathrm{g} / \mathrm{mL}$ and hs-CRP level $>3 \mathrm{mg} / \mathrm{L}$; (4) not receiving any form of vitamin $C$ supplementation within 3 months prior to the investigation. Patients with any one or more exclusion criteria were excluded from the investigation: (1) either hepatitis B surface antigen positive, hepatitis C antibody positive or HIV carrier; (2) acute infection within 1 month prior to the investigation; (3) neoplasm, hemopathy or active autoimmune disease; (4) use of steroids and/or immunosuppressive agents within 3 months prior to the investigation; (5) pregnancy or breast feeding.

In the present study, $128 \mathrm{MHD}$ patients were recruited from five dialysis facilities in North China. The mean age and the mean dialysis vintage of the patients were $64.1 \pm$ 12.1 years and $50.6 \pm 32.5$ [median 48 , inter-quartile range (IQR) 21, 72] months, respectively. Patients were divided into two groups as follows. In group $1(n=67)$, patients were orally administered with $200 \mathrm{mg} /$ day vitamin $C$ in the first 3 months, and then the vitamin $C$ supplementation was withdrawn in the next 3 months. In group 2 $(n=61)$, patients were not given vitamin $C$ in the first 3 months, and then they were orally administered with $200 \mathrm{mg} /$ day vitamin $C$ in the next 3 months. No patient was provided with omega-3 and/or vitamin E. Levels of plasma vitamin C, hs-CRP, prealbumin, albumin and biochemical parameters of interest were determined at the baseline and every 3 months throughout the study.

This study was approved by the Ethics Committee of Clinical Research, Peking University First Hospital (clinical trial number: NCT01356433). Written informed consent was obtained from all participants.

\section{Sample collection and laboratory measurements}

Fasting blood samples were collected from MHD patients through the arteriovenous fistula just before dialysis session. Collected blood samples were transported to the laboratory using heparin-containing tubes in an ice bath. Plasma was separated by centrifugation $(2,000 \mathrm{~g}, 10 \mathrm{~min})$ at $4^{\circ} \mathrm{C}$ within $30 \mathrm{~min}$. An aliquot of $200 \mu \mathrm{L}$ plasma was immediately mixed with $200 \mu \mathrm{L}$ of $10 \%$ metaphosphoric acid (MPA) due to the instability of vitamin $\mathrm{C}$ in plasma, and the mixture was then stored at $-80^{\circ} \mathrm{C}$ until further analysis within 2 weeks.

Vitamin $C$ level was determined by high-performance liquid chromatography (HPLC) (Agilent 1100 series, Agilent Technologies, USA) on a Diamonsil C18 column $(150 \mathrm{~mm} \times 4.6 \mathrm{~mm}, 3 \mu \mathrm{m})$ with UV detection according to the previously described method $[12,16]$. Intra-assay and inter-assay coefficients of variation were $2.7 \%$ and $2.5 \%$, respectively. The reference vitamin $\mathrm{C}$ level in normal population ranges from 4 to $14 \mu \mathrm{g} / \mathrm{mL}[17,18]$. 
EPO resistance index (ERI) was defined as the ratio of the dosage of recombinant human erythropoietin ( $\mathrm{rHuEpo}$ ) (IU/kg/week) and the concentration of hemoglobin $(\mathrm{g} / \mathrm{dl})$ on the day of plasma sampling. The hs-CRP level was determined using the immunonephelometric assay (First Chemical Pharmaceutical Co., Japan) with a detection limit of $0.1 \mathrm{mg} / \mathrm{L}$. Levels of prealbumin, albumin, ferritin, calcium, serum bicarbonate, uric acid and hemoglobin were determined by standard techniques in the Clinical Laboratory of Peking University First Hospital, Beijing, China.

Patient baseline demographics, including age, gender, primary cause of end stage renal disease (ESRD), dialysis vintage and drug use, were also collected and recorded.

\section{Statistical analysis}

Normally distributed variables, such as albumin, prealbumin and hemoglobin, were expressed as mean $\pm \mathrm{SD}$. Non-normally distributed variables, such as vitamin $C$ and hs-CRP, were presented as median and IQR. Levels of albumin, prealbumin and hemoglobin between groups were compared using Student's $t$-test or one-way analysis of variance (one-way-ANOVA). Categorical variables between groups were compared using $X^{2}$ test. Levels of vitamin $\mathrm{C}$ and hs-CRP between groups were compared using Mann-Whitney test or Kruskal Wallis Test. Statistical analysis was performed using SPSS version 11.5 (SPSS, Inc., Chicago, IL, USA). A P value of less than 0.05 was considered as statistically significant.

\section{Results}

\section{Demographics}

Among the 128 patients, 28 of them dropped out of the study before completion as follows: four heart failure, three acute infection, two repeated hemafecia, two pancytopenia of unknown reason, one renal transplant, one bladder carcinoma, five non-compliance, one wound and surgery, two transferred to other facilities and seven death. Consequently, a total of 100 patients (group 1: $n=48$; group 2: $\mathrm{n}=52$ ) were included in the final analysis (47 males, 53 females), with a mean age of $64.4 \pm 11.7$ years and a median dialysis vintage of 48 (IQR 21, 72) months (Table 1).

Among the 100 patients, the primary causes of ESRD were chronic glomerulonephritis $(n=20)$, interstitial nephropathy $(n=20)$, diabetic nephropathy $(n=20)$, hypertensive nephrosclerosis $(\mathrm{n}=18)$, polycystic disease $(\mathrm{n}=11)$, chronic pyelonephritis $(\mathrm{n}=3)$ and others $(\mathrm{n}=8)$. Table 1 lists the detailed demographics.

Patients in group 2 exhibited lower hs-CRP level, higher vitamin $\mathrm{C}$ and albumin levels compared with group 1. Moreover, there were more males in group 2. No statistical significance was found in terms of age,
Table 1 Baseline characteristics of the study population $(n=100)$

\begin{tabular}{|c|c|c|c|}
\hline Item & $\begin{array}{l}\text { Group } 1 \\
(n=48)\end{array}$ & $\begin{array}{l}\text { Group } 2 \\
(n=52)\end{array}$ & $p$ value \\
\hline Age (year) & $64.3 \pm 11.7$ & $64.4 \pm 11.8$ & 0.962 \\
\hline gender & & & 0.029 \\
\hline Male & $17(35.4 \%)$ & $30(57.7 \%)$ & \\
\hline female & $31(65.6 \%)$ & $22(42.3 \%)$ & \\
\hline BMI $\left(\mathrm{kg} / \mathrm{m}^{2}\right)$ & $21.9 \pm 3.3$ & $22.7 \pm 3.3$ & 0.248 \\
\hline KTN & $1.6 \pm 0.2$ & $1.5 \pm 0.3$ & 0.551 \\
\hline Dialysis vintage $(\mathrm{m})$ & $56.9 \pm 32.2$ & $44.8 \pm 32.0$ & 0.062 \\
\hline Diabetes & 9/39(23.1\%) & $11 / 41(21.2 \%)$ & 0.807 \\
\hline ACEI/ARB (case) & $6 / 48(12.5 \%)$ & $6 / 52(11.5 \%)$ & 1.000 \\
\hline Statins (case) & $2 / 48(4.2 \%)$ & $3 / 52(5.8 \%)$ & 1.000 \\
\hline BUN (mmol/l) & $25.2 \pm 5.6$ & $24.9 \pm 7.3$ & 0.884 \\
\hline Scr (umol/l) & $783.9 \pm 179.7$ & $920.1 \pm 256.9$ & 0.058 \\
\hline prealbumin (mg/l) & $295.6 \pm 86.6$ & $315.3 \pm 85.8$ & 0.256 \\
\hline albumin $(g / l)$ & $38.2 \pm 3.7$ & $40.0 \pm 4.2$ & 0.023 \\
\hline hemoglobin (g/l) & $107.2 \pm 16.6$ & $111.4 \pm 17.3$ & 0.217 \\
\hline ERI & $10.7 \pm 8.6$ & $9.3 \pm 6.1$ & 0.323 \\
\hline Ferritin (ug/l) & $417.8 \pm 266.5$ & $461.9 \pm 287.1$ & 0.429 \\
\hline \multirow[t]{2}{*}{ Vitamin C $(\mu \mathrm{g} / \mathrm{mL})$} & $1.5 \pm 0.8$ & $2.0 \pm 0.9$ & 0.003 \\
\hline & Median(IQR) & Median(IQR) & \\
\hline hsCRP(mg/l) & $9.6(6.0-13.8)$ & $6.2(4.2-11.0)$ & 0.005 \\
\hline
\end{tabular}

Note: age, BMI, dialysis vintage, BUN, Scr, prealbumin, albumin, hemoglobin, $\mathrm{ERI}$ and vitamin $C$ were presented as mean $\pm \mathrm{SD}$; hsCRP was presented as median and inter-quartile range (IQR)

Abbreviations: $B M I$ body mass index, $B U N$ blood urea nitrogen, $S c r$ serum creatinine, ERI EPO resistance index, $h s-C R P$ high hypersensitive $C$-reactive protein, IQR inter quartile range.

gender, body mass index, dialysis vintage, $\mathrm{Kt} / \mathrm{V}$, prealbumin, hemoglobin and ERI between two groups.

\section{Change of vitamin $\mathrm{C}$ level during the study}

At the baseline, the plasma vitamin $C$ level of all patients was less than $4 \mu \mathrm{g} / \mathrm{mL}$. However, this proportion was decreased to $20 \%$ after the vitamin C supplementation for 3 months.

For group 1, the vitamin $\mathrm{C}$ level was significantly increased at the end of the first 3 months $(p<0.001)$ compared with that at the baseline. After the vitamin $C$ supplementation was withdrawn, the vitamin $C$ level was significantly decreased at the end of the second 3 months compared with that at the end of the first 3 months $(p<0.001)$. Moreover, no significant difference in the vitamin $C$ level was found between the end of the second 3 months and the baseline $(p=0.606)$ (Figure 1).

For group 2, the vitamin $\mathrm{C}$ level remained unchanged at the end of the first 3 months $(\mathrm{p}=0.837)$ compared with that at the baseline. However, it was significantly increased 

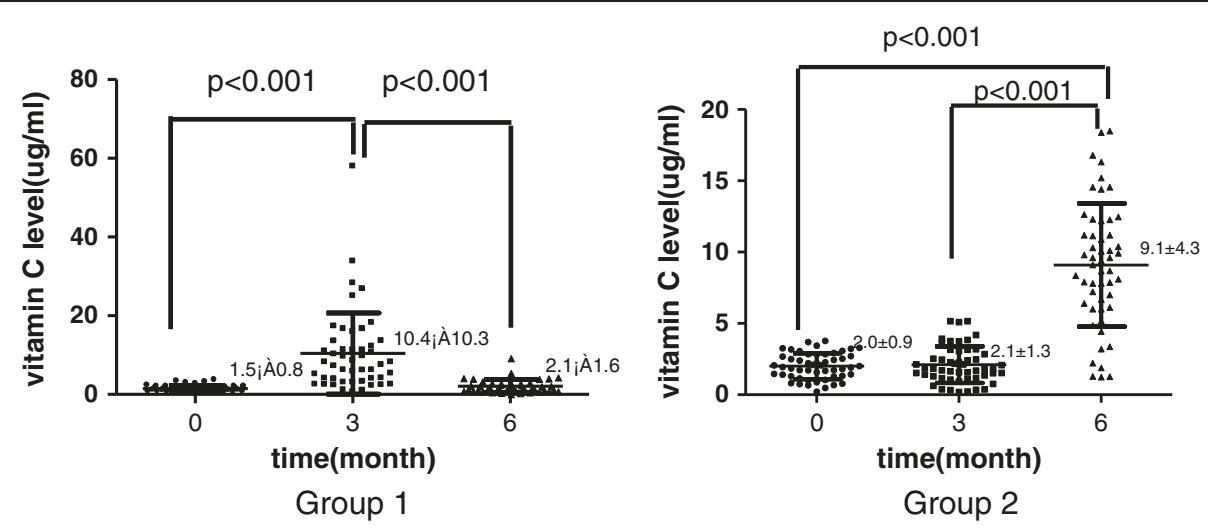

Figure 1 Influence of vitamin C supplementation on plasma vitamin C level. group1: patients were given oral vitamin C $200 \mathrm{mg}$ per day during the first 3 months and withdraw vitamin $C$ thereafter. group2: patients were given vitamin $C$ during the second 3 months; vitamin $C$ was presented as mean \pm SD; levels of vitamin C were compared among groups using one-way analysis of variance (1-way-ANOVA).

at the end of the second 3 months compared with that at the baseline $(\mathrm{p}<0.001)$ and the end of the first 3 months $(\mathrm{p}<0.001)$ (Figure 1).

\section{Change of hs-CRP level during the study}

For group 1, the hs-CRP level was significantly decreased at the end of the first 3 months $(\mathrm{p}<0.001)$ compared with that at the baseline. After the vitamin $C$ supplementation was withdrawn, the hs-CRP level was significantly increased at the end of the second 3 months compared with that at the end of the first 3 months $(\mathrm{p}=0.014)$. Moreover, no significant difference in the hs-CRP level was found between the end of the second 3 months and the baseline $(\mathrm{p}=0.106)$ (Figure 2).

For group 2, the hs-CRP level remained unchanged at the end of the first 3 months $(\mathrm{p}=0.663)$ compared with that at the baseline. However, it was significantly decreased at the end of the second 3 months compared with that at the baseline $(p=0.005)$ and the end of the first 3 months $(\mathrm{p}<0.001)$ (Figure 2).

\section{Change of prealbumin and albumin levels during the study}

For group 1, levels of prealbumin and albumin exhibited a slight increase $(p>0.05)$ at the end of the first 3 months compared with those at the baseline. After the vitamin $\mathrm{C}$ supplementation was withdrawn, levels of prealbumin and albumin $(p>0.05)$ were decreased at the end of the second 3 months (Table 2).

For group 2, levels of prealbumin and albumin remained unchanged at the end of the first 3 months $(\mathrm{p}>0.05)$ compared with those at the baseline. However, a significant increase in prealbumin $(\mathrm{p}=0.018)$ and an increase trend in albumin $(\mathrm{p}>0.05)$ were observed at the end of the second 3 months compared with those at the end of the first 3 months (Table 2).

\section{Change of ERI during the study}

For group 1, a decrease trend in ERI, ferritin and EPO dosage (all p > 0.05) and an increase trend in hemoglobin ( $p>0.05)$ were observed at the end of the first 3 months
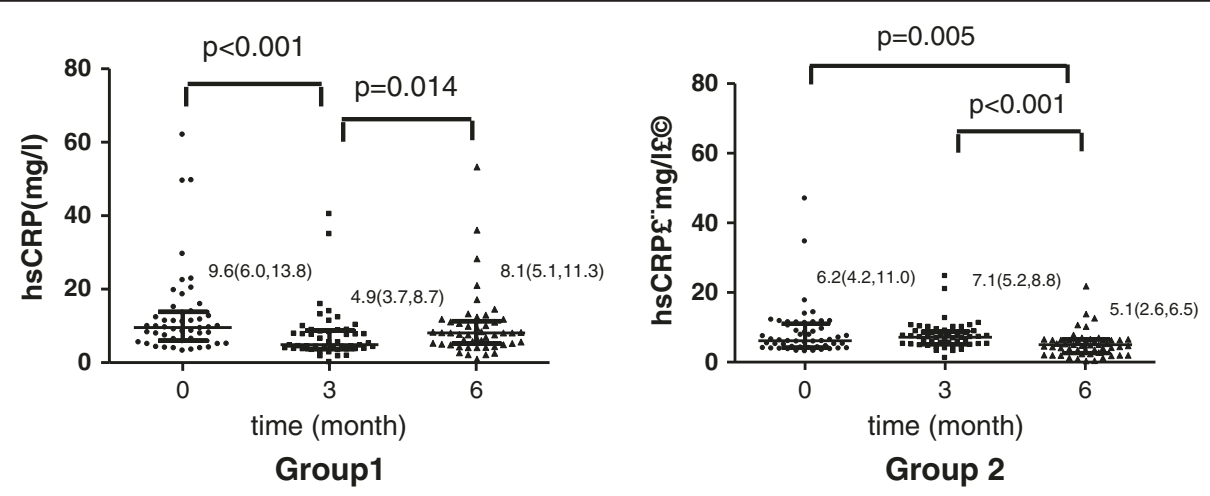

Figure 2 Effect of vitamin C supplementation on plasma hs-CRP level. Group 1: patients were orally administered with $200 \mathrm{mg} / \mathrm{day}$ vitamin $C$ during the first 3 months, and the vitamin $C$ administration was withdrawn thereafter. Group 2: patients were administered with vitamin $C$ during the second 3 months. Hs-CRP level was presented as Median (IQR); levels of hs-CRP were compared among groups using Kruskal Wallis Test. 
Table 2 Effect of vitamin C supplementation on parameters during 6 months $(n=100)$

\begin{tabular}{|c|c|c|c|c|c|c|}
\hline \multirow[t]{2}{*}{ Item } & \multicolumn{3}{|c|}{ Group $1(n=48)$} & \multicolumn{3}{|c|}{ Group $2(n=52)$} \\
\hline & Baseline & Month 3 & Month 6 & Baseline & Month 3 & Month 6 \\
\hline prealbumin (mg/l) & $295.6 \pm 86.6$ & $296.7 \pm 60.1$ & $272.1 \pm 69.3$ & $315.3 \pm 85.8$ & $302.9 \pm 60.3$ & $336.9 \pm 69.5^{a}$ \\
\hline albumin (g/l) & $38.2 \pm 3.7$ & $38.3 \pm 3.1$ & $37.6 \pm 2.6$ & $40.0 \pm 4.2$ & $39.6 \pm 2.8$ & $40.4 \pm 2.4$ \\
\hline hemoglobin (g/l) & $107.2 \pm 16.6$ & $109.9 \pm 14.1$ & $109.3 \pm 14.2$ & $111.4 \pm 17.3$ & $110.9 \pm 20.4$ & $111.9 \pm 25.4$ \\
\hline ERI & $10.7 \pm 8.6$ & $8.3 \pm 6.6$ & $9.1 \pm 7.4$ & $9.3 \pm 6.1$ & $8.6 \pm 6.7$ & $7.5 \pm 6.4$ \\
\hline Ferritin (ug/l) & $417.8 \pm 266.5$ & $355.0 \pm 256.4$ & $444.2 \pm 333.5$ & $461.9 \pm 287.1$ & $500.0 \pm 314.2$ & $445.9 \pm 352.7$ \\
\hline \multirow[t]{2}{*}{ Vitamin C $(\mu \mathrm{g} / \mathrm{mL})$} & \multirow[t]{2}{*}{$1.5 \pm 0.8$} & $10.4 \pm 10.3^{b}$ & \multirow[t]{2}{*}{$2.1 \pm 1.6^{c}$} & \multirow[t]{2}{*}{$2.0 \pm 0.9$} & $2.1 \pm 1.3$ & \multirow[t]{2}{*}{$9.1 \pm 4.3^{b c}$} \\
\hline & & Median(IQR) & & & Median(IQR) & \\
\hline rHuEpo (x $10^{3} \mathrm{U} /$ week) & $6.0(3.0-9.0)$ & $4.5(2.8-6.0)$ & $5.2(2.6-8.8)$ & $6.0(4.0-8.8)$ & $6.0(3.0-8.8)$ & $4.2(2.7-6.0)$ \\
\hline hsCRP (mg/l) & $9.6(6.0-13.8)$ & $4.9(3.7-8.7)^{b}$ & $8.1(5.1-11.3)^{d}$ & $6.2(4.2-11.0)$ & $7.1(5.2-8.8)$ & $5.1(2.6-6.5)^{b c}$ \\
\hline
\end{tabular}

Note: ${ }^{a} p<0.05$ :compared with group baseline; ${ }^{b} p<0.01$ :compared with group baseline; ${ }^{c} p<0.01$ :compared with group Month 3; ${ }^{d} p<0.05$ :compared with group Month 3; prealbumin, albumin, hemoglobin, ERI, ferritin and vitamin C levels were presented as mean \pm SD; EPO dosage and hs-CRP level were presented as median and inter-quartile range (IQR).

Abbreviations: ERI EPO resistance index, rHuEpo, recombinant human erythropoietin, $h s-C R P$ high hypersensitive $C$-reactive protein, IQR inter quartile range.

compared with those at the baseline. At the end of the second 3 months, ERI, ferritin and EPO dosage (all $\mathrm{p}>0.05$ ) were increased without statistical significance compared with those at the end of the first 3 months, whereas the hemoglobin level remained unchanged at the end of the second 3 months (Table 2).

For group 2, a decrease trend in ERI and hemoglobin and an increase trend in ferritin were observed at the end of the first 3 months compared with those at the baseline (all $p>0.05$ ), whereas the EPO dosage remained unchanged. At the end of the second 3 months, a decrease trend in ERI, ferritin and EPO dosage and an increase trend in hemoglobin were observed (all p >0.05) compared with those at the end of the first 3 months (Table 2).

\section{Discussion}

In the present study, we showed that the plasma hsCRP level in MHD patients could be reduced by oral vitamin $C$ supplementation. The proportion of patients with a plasma vitamin $C$ level of less than $4 \mu \mathrm{g} / \mathrm{mL}$ was decreased to $20 \%$ after the vitamin $C$ supplementation for 3 months. We also found an increase trend in plasma prealbumin level after the vitamin $C$ supplementation. In addition, a better plasma albumin, hemoglobin, EPO dosage and ERI response to vitamin $C$ supplementation was observed without statistical significance.

Previous study demonstrated that MHD patients have remarkably low plasma vitamin $C$ levels, frequently $<10 \mu \mathrm{M}$, even $<2 \mu \mathrm{M}[8,19]$. In our previous study, a plasma vitamin C level of $<4 \mu \mathrm{g} / \mathrm{mL}(22.8 \mu \mathrm{mol} / \mathrm{L})$ is presented in $64.4 \%$ dialysis patients [12]. In our current study, $20 \%$ patients still exhibited a persistent low plasma vitamin $C$ level after the vitamin $C$ supplementation for 3 months, suggesting that an individualized dosage of vitamin $C$ supplementation should be considered.
Low-level, persistent inflammation is prevalent in MHD patients, although there is no convincing evidence of systemic or restricted infection in clinical practice. Vitamin C deficiency is caused by inadequate dietary intake, loss during dialysis procedure, impaired metabolism and reduced tubular reabsorption [7,10,20-22].

Miyata and Wang S. et al. observed that the concentration of in vitro plasma ascorbic acid in uremic patients is decreased more rapidly $(0.16 \%$ per $\mathrm{min})$ than that in normal subjects $(0.09 \%$ per $\mathrm{min})$ [23,24]. This finding suggested that the uremic plasma consumes more vitamin C than healthy plasma, which may be related to excessive toxin retention and metabolic acidosis [25]. In vivo, the volume overload [26] and bio-incompatibility of dialysis materials and non-sterile dialysate may also contribute to the inflammatory status [27].

In our previous cross-sectional study, we found that a negative correlation existed between the plasma vitamin $\mathrm{C}$ level and inflammation status in MHD patients [12]. We hypothesized that vitamin $\mathrm{C}$, as an electron donor, had anti-oxidative effects, and its oral supplementation could improve the inflammatory status in MHD patients. Tarng D C et al. [28] reported that the 8-OHdG level of cellular DNA, as an evaluative indicator of oxidative DNA damage in reactive oxygen species-mediated diseases [15], is reduced after the vitamin $C$ supplementation for 8 weeks in chronic hemodialysis patients. However, this beneficial effect in MHD patients has not been reported by other studies. In Fumeron's study [13], 33 MHD patients were orally administered with $250 \mathrm{mg}$ vitamin $\mathrm{C}$ thrice weekly after each dialysis session for 2 months, and no evident improvement is observed in oxidative/ anti-oxidative stress and inflammation markers. Kamgar $\mathrm{M}$ et al. [14] reported a decrease trend in CRP level after an oral supplementation of $250 \mathrm{mg} /$ day vitamin C for 2 months in 20 MHD patients. In our present study, 
the hs-CRP level was decreased by oral supplementation of $200 \mathrm{mg}$ /day vitamin $\mathrm{C}$ in both groups, and the hs-CRP level was increased again after the vitamin $C$ supplementation was withdrawn in group 1 . Unlike other inconclusive results from previous studies, we showed that the vitamin $\mathrm{C}$ supplementation doubtlessly had a beneficial effect. Our results were more convincing due to following advantages: (1) relative larger sample size; (2) relative longer period of observation; (3) randomized controlled cross-over design; (4) more importantly, selected patients were with low vitamin $\mathrm{C}$ level and high hs-CRP level, and this patient population might respond well to inflammation-induced vitamin $C$ consumption. In this study, several patients took anti-inflammatory drugs, such as ACEI/ARB, statins, but remain unchanged during the study period. Therefore, the anti-inflammatory effects of these drugs on our patients could be sagely ignored.

Recent evidence showed that the plasma vitamin C level is positively associated with levels of hemoglobin [29], albumin [30] and prealbumin [12], and negatively associated with ERI [31-33]. After 6 months of vitamin C supplementation, levels of prealbumin, albumin and hemoglobin are significantly increased in the preliminary study. In the present randomized controlled cross-over study, we also found beneficial responses of these markers upon the vitamin C supplementation, but statistically insignificant, which could be due to the long half-life of serum albumin and hemoglobin, and the short interventional duration. These beneficial effects might be caused by anti-oxidative effect of vitamin C.

Consistent with our data, previous study showed that the vitamin $C$ supplementation improves the responsiveness to EPO in hemodialysis patients with refractory anemia and hyperferritinemia [31]. In our present study, a decrease trend in ERI, ferritin and EPO dosage, and an increase trend in hemoglobin were observed after the oral vitamin $C$ supplementation for 3 months. One possible mechanism for this effect might be the electron offering ability of vitamin $\mathrm{C}$. Vitamin $\mathrm{C}$ mobilizes storage iron by reducing ferric iron $\left(\mathrm{Fe}^{+3}\right)$ to ferrous iron $\left(\mathrm{Fe}^{+2}\right)$, including the portion of tissue iron as hemosiderin [34], leading to an increased bioavailability of iron and improved red blood cell production. In the present study, the improvement in hemoglobin was associated with significantly decreased hs-CRP levels during the vitamin C supplementation, but not in controls, which might be due to the anti-oxidative ability of vitamin $C$.

In this study, the prealbumin concentration was significantly increased after the oral vitamin $\mathrm{C}$ administration in group 2 but not in group 1, and ERI was decreased in group 2 even in without-drug phase. Therefore, some other important factors, in addition to the relatively short interventional duration, were probably not included in the present investigation.
In order to minimize the possible accumulation of oxalate in patients, the dosage of vitamin $\mathrm{C}$ was selected as $200 \mathrm{mg} /$ day. Dosages as high as 500 to $1,000 \mathrm{mg} /$ day for 3 or more than 3 weeks induce significantly increased plasma oxalate levels $[35,36]$. Our present study had some limitations as follows. (1) The duration of the intervention was relatively short, although changes in hs-CRP level were observed. Changes of albumin, prealbumin, hemoglobin, EPO dosages and ERI were not significant due to their relatively longer half-life, because duration of 3 months only permitted one red blood cell life-span to reach steady state [37]. (2) A total of 28 (21.9\%) patients dropped out during the observation, which might result in the imbalance of parameters between the two groups. (3) This investigation did not contain the placebo in the control group.

\section{Conclusion}

In conclusion, our cross-over study indicated that the inflammatory status in MHD patients with plasma vitamin C deficiency and high levels of inflammatory markers could be partially improved by long-term oral administration of small doses of vitamin C. A multi-center randomized controlled study with relatively larger sample size is required to confirm the role of vitamin $\mathrm{C}$ in improving the microinflammatory state in MHD patients. Moreover, further study is also necessary to assess the long-term outcome of oral vitamin $C$ supplementation in MHD patients.

\section{Competing interests}

The authors declare that they have no competing interests.

\section{Authors' contributions}

ZKY participated in the design of the study, sampling procedure, and drafted the manuscript. LYH, CXY, LL, BWY, GWY and WLY participated in the design of the study and sampling procedure. ZL conceived the study, and participated in its design and coordination and performed statistical analysis. All authors read and approved the final manuscript.

\section{Acknowledgements}

The authors are grateful to Professor Geng Qiuming, Central Lab, Peking University Third Hospital, for sampling procedure and statistical advice.

\section{Author details}

'Department of Medicine, Renal Division, Peking University First Hospital, Beijing, P. R. China. ${ }^{2}$ Peking University Institute of Nephrology, Beijing, P. R. China. ${ }^{3}$ Key Laboratory of Renal Disease, Ministry of Health of China, Beijing, P. R. China. ${ }^{4}$ Department of Nephrology, Peking University People's Hospital, Beijing, P. R. China. ${ }^{5}$ Jilin University Fourth Hospital, Changchun, Jilin, P. R. China. ${ }^{6}$ Beijing Daxing district People's Hospital, Beijing, P. R. China.

${ }^{7}$ Guangwai Hospital, Beijing, P. R. China. ${ }^{8}$ Beijing No. 2 Hospital, Beijing, P. R. China.

Received: 10 November 2012 Accepted: 5 November 2013 Published: 14 November 2013

\section{References}

1. Foley RN, Parfrey PS, Sarnak MJ: Clinical epidemiology of cardiovascular disease in chronic renal disease. Am J Kidney Dis 1998, 32(5 Suppl 3):S112-S119. 
2. Yeun JY, Levine RA, Mantadilok V, Kaysen GA: C-reactive protein predicts all-cause and cardiovascular mortality in hemodialysis patients. Am J Kidney Dis 2000, 35(3):469-476.

3. Zoccali C: Cardiovascular risk in uraemic patients-is it fully explained by classical risk factors? Nephrol Dial Transplant 2000, 15(4):454-457.

4. Owen WF, Lowrie EG: C-reactive protein as an outcome predictor for maintenance hemodialysis patients. Kidney Int 1998, 54(2):627-636.

5. Ridker PM, Cushman M, Stampfer MJ, Tracy RP, Hennekens $\mathrm{CH}$ : Inflammation, aspirin, and the risk of cardiovascular disease in apparently healthy men. N Engl J Med 1997, 336(14):973-979.

6. Zimmermann J, Herrlinger S, Pruy A, Metzger T, Wanner C: Inflammation enhances cardiovascular risk and mortality in hemodialysis patients. Kidney Int 1999, 55(2):648-658.

7. Morena M, Cristol JP, BosC JY, Tetta C, Forret G, Leger CL, Delcourt C, Papoz L, Descomps B, Canaud B: Convective and diffusive losses of vitamin $C$ during haemodiafiltration session: a contributive factor to oxidative stress in haemodialysis patients. Nephrol Dial Transplant 2002, 17(3):422-427

8. Wang S, Eide TC, Sogn EM, Berg KJ, Sund RB: Plasma ascorbic acid in patients undergoing chronic haemodialysis. Eur J Clin Pharmacol 1999, 55(7):527-532.

9. Washio K, Inagaki M, Tsuji M, Morio Y, Akiyama S, Gotoh H, Gotoh T, Gotoh $\mathrm{Y}$, Oguchi K: Oral vitamin C supplementation in hemodialysis patients and its effect on the plasma level of oxidized ascorbic acid and $\mathrm{Cu} / \mathrm{Zn}$ superoxide dismutase, an oxidative stress marker. Nephron Clin Pract 2008, 109(2):c49-c54

10. Sullivan JF, Eisenstein $A B$ : Ascorbic acid depletion during hemodialysis. JAMA 1972, 220(13):1697-1699.

11. Paniagua $R$, Frias $Y$, de Ventura MJ, Rodriguez E, Hurtado ME, Alcantara G, Vazquez R, Ortiz R, Salcedo M, Rios ME, et al: C-reactive protein and anti-Chlamydia pneumoniae antibodies as risk factors of cardiovascular death in incident patients on peritoneal dialysis. Perit Dial Int 2003, 23(2):132-137.

12. Zhang K, Liu L, Cheng X, Dong J, Geng Q, Zuo L: Low levels of vitamin C in dialysis patients is associated with decreased prealbumin and increased C-reactive protein. BMC Nephrol 2011, 12:18.

13. Fumeron C, Nguyen-Khoa T, Saltiel C, Kebede M, Buisson C, Drueke TB, Lacour B, Massy ZA: Effects of oral vitamin C supplementation on oxidative stress and inflammation status in haemodialysis patients. Nephrol Dial Transplant 2005, 20(9):1874-1879.

14. Kamgar M, Zaldivar F, Vaziri ND, Pahl MV: Antioxidant therapy does not ameliorate oxidative stress and inflammation in patients with end-stage renal disease. J Natl Med Assoc 2009, 101(4):336-344.

15. Kasai H: Analysis of a form of oxidative DNA damage, 8-hydroxy-2'deoxyguanosine, as a marker of cellular oxidative stress during carcinogenesis. Mutat Res 1997, 387(3).

16. Zhang K, Dong J, Cheng X, Bai W, Guo W, Wu L, Zuo L: Association between vitamin $C$ deficiency and dialysis modalities. Nephrology (Carlton) 2012, 17(5):452-457

17. Reuler JB, Broudy VC, Cooney TG: Adult scurvy. JAMA 1985, 253(6):805-807.

18. Richter A, Kuhlmann MK, Seibert E, Kotanko P, Levin NW, Handelman GJ: Vitamin $C$ deficiency and secondary hyperparathyroidism in chronic haemodialysis patients. Nephrol Dial Transplant 2008, 23(6):2058-2063.

19. Jackson P, Loughrey CM, Lightbody JH, McNamee PT, Young IS: Effect of hemodialysis on total antioxidant capacity and serum antioxidants in patients with chronic renal failure. Clin Chem 1995, 41 (8 Pt 1):1135-1138.

20. Alkhunaizi AM, Chan L: Secondary oxalosis: a cause of delayed recovery of renal function in the setting of acute renal failure. J Am Soc Nephrol 1996, 7(11):2320-2326

21. Durose $\mathrm{CL}$, Holdsworth M, Watson V, Przygrodzka F: Knowledge of dietary restrictions and the medical consequences of noncompliance by patients on hemodialysis are not predictive of dietary compliance. J Am Diet Assoc 2004, 104(1):35-41.

22. Boeschoten EW, Schrijver J, Krediet RT, Schreurs WH, Arisz L: Deficiencies of vitamins in CAPD patients: the effect of supplementation. Nephrol Dial Transplant 1988, 3(2):187-193.

23. Wang S, Schram IM, Sund RB: Determination of plasma ascorbic acid by HPLC: method and stability studies. Eur J Pharm Sci 1995, 3(4):231-239.

24. Miyata T, Wada Y, Cai Z, lida Y, Horie K, Yasuda Y, Maeda K, Kurokawa K, van Ypersele DSC: Implication of an increased oxidative stress in the formation of advanced glycation end products in patients with end-stage renal failure. Kidney Int 1997, 51(4):1170-1181.

25. Bellocq A, Suberville S, Philippe C, Bertrand F, Perez J, Fouqueray B, Cherqui $\mathrm{G}$, Baud L: Low environmental $\mathrm{pH}$ is responsible for the induction of nitric-oxide synthase in macrophages. Evidence for involvement of nuclear factor-kappaB activation. J Biol Chem 1998, 273(9):5086-5092.

26. Demirci MS, Demirci C, Ozdogan O, Kircelli F, Akcicek F, Basci A, Ok E, Ozkahya M: Relations between malnutrition-inflammation-atherosclerosis and volume status. The usefulness of bioimpedance analysis in peritoneal dialysis patients. Nephrol Dial Transplant 2011, 26(5):1708-1716.

27. Stenvinkel $P$, Alvestrand A: Inflammation in end-stage renal disease: sources, consequences, and therapy. Semin Dial 2002, 15(5):329-337.

28. Tarng DC, Liu TY, Huang TP: Protective effect of vitamin C on 8-hydroxy2'-deoxyguanosine level in peripheral blood lymphocytes of chronic hemodialysis patients. Kidney Int 2004, 66(2):820-831.

29. Finkelstein FO, Juergensen $P$, Wang $S$, Santacroce $S$, Levine M, Kotanko P Levin NW, Handelman GJ: Hemoglobin and plasma vitamin C levels in patients on peritoneal dialysis. Perit Dial Int 2011, 31(1):74-79.

30. Lee EJ, Myint CC, Tay ME, Yusuf N, Ong CN: Serum ascorbic acid and protein calorie malnutrition in continuous ambulatory peritoneal dialysis patients. Adv Perit Dial 2001, 17:219-222.

31. Attallah N, Osman-Malik Y, Frinak S, Besarab A: Effect of intravenous ascorbic acid in hemodialysis patients with EPO-hyporesponsive anemia and hyperferritinemia. Am J Kidney Dis 2006, 47(4):644-654.

32. Deicher R, Ziai F, Habicht A, Bieglmayer C, Schillinger M, Horl WH: Vitamin C plasma level and response to erythropoietin in patients on maintenance haemodialysis. Nephrol Dial Transplant 2004, 19(9):2319-2324.

33. Keven K, Kutlay S, Nergizoglu G, Erturk S: Randomized, crossover study of the effect of vitamin C on EPO response in hemodialysis patients. Am J Kidney Dis 2003, 41(6):1233-1239.

34. Smith $\mathrm{CH}$, Bidlack WR: Interrelationship of dietary ascorbic acid and iron on the tissue distribution of ascorbic acid, iron and copper in female guinea pigs. J Nutr 1980, 110(7):1398-1408.

35. Tomson CR, Channon SM, Parkinson IS, McArdle P, Qureshi M, Ward MK, Laker MF: Correction of subclinical ascorbate deficiency in patients receiving dialysis: effects on plasma oxalate, serum cholesterol, and capillary fragility. Clin Chim Acta 1989, 180(3):255-264.

36. Pru C, Eaton J, Kjellstrand C: Vitamin C intoxication and hyperoxalemia in chronic hemodialysis patients. Nephron 1985, 39(2):112-116.

37. Bienfait HF, van den Briel ML: Rapid mobilization of ferritin iron by ascorbate in the presence of oxygen. Biochim Biophys Acta 1980, 631(3):507-510.

doi:10.1186/1471-2369-14-252

Cite this article as: Zhang et al:: Cross-over study of influence of oral vitamin C supplementation on inflammatory status in maintenance hemodialysis patients. BMC Nephrology 2013 14:252.

\section{Submit your next manuscript to BioMed Central and take full advantage of:}

- Convenient online submission

- Thorough peer review

- No space constraints or color figure charges

- Immediate publication on acceptance

- Inclusion in PubMed, CAS, Scopus and Google Scholar

- Research which is freely available for redistribution 